\title{
Cerebral metabolism in streptozotocin-diabetic rats: an in vivo magnetic resonance spectroscopy study
}

\author{
G.J. Biessels ${ }^{1}$, K.P.J. Braun ${ }^{2}$, R.A. de Graaf ${ }^{3}$, P. van Eijsden ${ }^{3}$, W.H. Gispen ${ }^{1}$, K. Nicolay ${ }^{3}$ \\ ${ }^{1}$ Department of Medical Pharmacology, Rudolf Magnus Institute for Neurosciences, University Medical Center Utrecht, \\ The Netherlands \\ ${ }^{2}$ Department of Neurology, Rudolf Magnus Institute for Neurosciences, University Medical Center Utrecht, The Netherlands \\ ${ }^{3}$ Department of in vivo NMR, Bijvoet Center for Biomolecular Research, Utrecht University, The Netherlands
}

\section{Abstract}

Aims/hypothesis. It is increasingly evident that the brain is another site of diabetic end-organ damage. The pathogenesis has not been fully explained, but seems to involve an interplay between aberrant glucose metabolism and vascular changes. Vascular changes, such as deficits in cerebral blood flow, could compromise cerebral energy metabolism. We therefore examined cerebral metabolism in streptozotocin-diabetic rats in vivo by means of localised ${ }^{31} \mathrm{P}$ and ${ }^{1} \mathrm{H}$ magnetic resonance spectroscopy.

Methods. Rats were examined 2 weeks and 4 and 8 months after diabetes induction. A non-diabetic group was examined at baseline and after 8 months. Results. In ${ }^{31} \mathrm{P}$ spectra the phosphocreatine:ATP, phosphocreatine:inorganic phosphate and ATP:inorganic phosphate ratios and intracellular $\mathrm{pH}$ in diabetic rats were similar to controls at all time points. In ${ }^{1} \mathrm{H}$ spectra a lactate resonance was detected as frequently in controls as in diabetic rats. Compared with baseline and 8-month controls ${ }^{1} \mathrm{H}$ spectra did, however, show a statistically significant decrease in $\mathrm{N}$-acetylaspartate:total creatine $(-14 \%$ and $-23 \%)$ and $N$ acetylaspartate:choline $(-21 \%$ and $-17 \%)$ ratios after 2 weeks and 8 months of diabetes, respectively. Conclusion/interpretation. No statistically significant alterations in cerebral energy metabolism were observed after up to 8 months of streptozotocin-diabetes. These findings indicate that cerebral blood flow disturbances in diabetic rats do not compromise the energy status of the brain to a level detectable by magnetic resonance spectroscopy. Reductions in $\mathrm{N}$ acetylaspartate levels in the brain of STZ-diabetic rats were shown by ${ }^{1} \mathrm{H}$ spectroscopy, which could present a marker for early metabolic or functional abnormalities in cerebral neurones in diabetes. [Diabetologia (2001) 44: 346-353]

Keywords Magnetic resonance spectroscopy, energy metabolism, phosphocreatine, $N$-acetylaspartate, complications, brain, diabetes mellitus, pathophysiology, experimental.
It is becoming increasingly clear that the brain is another site of diabetic end-organ damage [1]. Some diabetic patients develop cognitive deficits, which are

Received: 3 August 2000 and in revised form: 17 October 2000

Corresponding author: G.-J. Biessels, PhD, HPN G03.228, Department of Neurology, University Medical Centre Utrecht, PO Box 85500, 3508GA Utrecht, The Netherlands

Abbreviations: Cho, Choline-containing compounds; MRS, magnetic resonance spectroscopy; NAA, $N$-acetylaspartate; $\mathrm{PCr}$, phosphocreatine ; $\mathrm{Pi}$, inorganic phosphate; STZ, streptozotocin; tCr, total creatine; NMR, nuclear magnetic resonance. generally moderate in young adults [2] but can be more pronounced in the elderly [3]. Recent epidemiological studies even report an association between diabetes and dementia $[3,4]$. Given the prevalence of diabetes among the elderly and the effect of cognitive impairment and dementia on the quality of life of patients and health care resources, a better understanding of the effects of diabetes on the brain is needed.

Experimentally diabetic rodents develop cerebral deficits similar to those observed in diabetic patients. Streptozotocin (STZ)-diabetic rats have been found to have impaired learning and memory functions [5] and increases in the peak latencies of brainstem audi- 
tory and visual evoked potentials [6, 7] and morphological alterations [8]. The development of these deficits is related to diabetes duration and the severity of hyperglycaemia [6]. Because insulin treatment prevents the deficits [9], they must be due to hyperglycaemia and insulin deficiency, rather than a direct toxic effect of STZ on the brain. Moreover, STZ transport and cytotoxicity is dependent on the GLUT-2 glucose transporter [10], which is not expressed at the blood-brain barrier in rats [11].

The pathogenesis of cerebral disorders in diabetes is incompletely understood. It seems to be a multifactorial process involving the adverse effects of chronic hyperglycaemia, and probably also those of recurrent hypoglycaemia, on the brain [1]. Analogous to the pathogenesis of peripheral diabetic neuropathy, chronic hyperglycaemia can lead to both metabolic and vascular disturbances in the brain. Cerebrovascular changes can be found in both diabetic rodents and patients. Studies in STZ-diabetic rats have shown regional reductions in cerebral blood flow within weeks $[12,13]$ to months [14] after diabetes induction. Moreover, thickening of capillary basement membranes [15] and shortening of the length of the capillary network in the neocortex have been noted [8]. Studies of diabetic patients report regional decreases in cerebral blood flow and impaired cerebrovascular reactivity as well as structural alterations in the cerebral vasculature, including thickening of capillary basement membranes [16]. These functional and structural changes in the vasculature could impede the delivery of nutrients and oxygen to the brain, thus affecting cerebral energy metabolism. This issue has been addressed in a number of ex vivo biochemical studies in diabetic rats but the results have been inconsistent, possibly partially due to interference of post-mortem changes. Concentrations of lactate were found to be unaltered $[17,18]$ or slightly increased [19], those of ATP unaltered [17, 19] or slightly decreased [18] and those of phosphocreatine (PCr) unaltered [17, 18] or increased [19].

We examined cerebral metabolism in STZ-diabetic rats longitudinally in vivo using ${ }^{31} \mathrm{P}$ and ${ }^{1} \mathrm{H}$ magnetic resonance spectroscopy (MRS). With ${ }^{31} \mathrm{P}$ MRS the relative concentrations of PCr, ATP and inorganic phosphate $(\mathrm{Pi})$ can be measured, reflecting the energy status of the brain [20]. In addition, the intracellular $\mathrm{pH}\left(\mathrm{pH}_{\mathrm{i}}\right)$ can be estimated [20]. With ${ }^{1} \mathrm{H}$ MRS increased lactate concentrations, as a consequence of increased anaerobic glycolysis, can be detected. In addition, ${ }^{1} \mathrm{H}$ MRS yields a measure of the levels of $N$-acetylaspartate (NAA), total creatine (tCr; the sum of creatine and phosphocreatine) and cholinecontaining compounds (Cho; including compounds such as choline, acetylcholine and phosphorylcholine) in the brain. The measurement of these metabolites provides information on neuronal integrity and membrane phospholipid metabolism [20, 21].

\section{Materials and methods}

Animals. Male Wistar rats (starting weight $\sim 350 \mathrm{~g}$, aged 3 months, UWU-CPD, Harlan, Utrecht, The Netherlands) were housed on sawdust, maintained on a $12 \mathrm{~h}-12 \mathrm{~h}$ light-dark cycle and given free access to food and water. The rats were weighed weekly. Diabetes was induced by a single intravenous injection of streptozotocin (Serva Feinbiochemica, Heidelberg, Germany) at a dose of $30 \mathrm{mg} / \mathrm{kg}$ body weight, dissolved in saline. Blood glucose was measured 4 days after the STZ injection in blood samples obtained by tail prick, by a strip-operated blood glucose sensor (Companion2, Medisense Ltd, Birmingham, UK). Blood glucose concentrations were higher than $15.0 \mathrm{mmol} / \mathrm{l}$ in all STZ-injected animals. We did not measure ketone bodies but previous studies in this model have shown that at STZ dosages up to $50 \mathrm{mg} / \mathrm{kg}$ animals become only mildly ketotic $[19,22,23]$. Given the relative abundance of glucose as an energy source in the brain of STZ-diabetic rats, these low concentrations of ketone bodies should not affect the cerebral metabolites that were the subject of our study. The principles of laboratory animal care were followed and the Utrecht University Committee for welfare of experimental animals approved the experiments.

Experimental design. We used two groups of rats. The first consisted of eight non-diabetic rats, which were examined at baseline and after 8 months. The second consisted of seven diabetic rats. In these rats nuclear magnetic resonance (NMR) measurements were done at 2 weeks, 4 and 8 months after induction of diabetes. The NMR protocol included $\mathrm{T}_{2}$ weighted ${ }^{1} \mathrm{H}$ MR imaging, single voxel ${ }^{1} \mathrm{H}$ MRS and ${ }^{31} \mathrm{P}$ MRS. One control and one diabetic rat did not survive the 8 months of the experiment. Spare animals replaced these rats in the final measurements.

Nuclear magnetic resonance (NMR) protocol. Nuclear magnetic resonance experiments were done on a SIS Co spectrometer (Varian Associates, Palo Alto, Calif., USA) interfaced to a 4.7 T Oxford magnet (Oxford Instruments Ltd., Oxford, UK), equipped with actively shielded gradients with a maximum gradient strength of $32 \mathrm{mT} / \mathrm{m}$. For ${ }^{1} \mathrm{H}$ NMR measurements, a saddle-shaped volume coil was used for radio frequency pulse transmission. An inductively coupled surface coil $(2 \mathrm{~cm}$ diameter) was placed against the skull and used for signal detection. For ${ }^{31} \mathrm{P}$ MRS experiments, a two turn surface coil $(2.5 \mathrm{~cm}$ diameter) was used for both signal transmission and detection.

The rats were anaesthetised by subcutaneous injection of a mixture of fluanisone $(5.5 \mathrm{mg} / \mathrm{kg})$, fentanylcitrate $(0.17 \mathrm{mg} /$ $\mathrm{kg}$ ) and midazolam $(2.75 \mathrm{mg} / \mathrm{kg})$, followed by intubation and mechanical ventilation with $0.7-0.8 \%$ halothane in $\mathrm{O}_{2} \mathrm{~N}_{2} \mathrm{O}$ $(30 / 70 \%)$ throughout the NMR protocol, which lasted approximately $3 \mathrm{~h}$. Body temperature was continuously measured and kept within the physiological range, using a heating pad with circulating water. Expiratory $\mathrm{CO}_{2}$ was monitored (Datascope, Paramus, N.J., USA) and used to assess and adjust the depth of anaesthesia. Average end-expiratory $\mathrm{CO}_{2}$ levels were similar among the experimental groups at the start of the NMR protocol and did not change appreciably during the measurements. Respiratory volumes and rates were based on previous experiments in which arterial blood gas was sampled. We did not analyse blood gas or monitor arterial pressure in the present experiments because cannulation of the femoral arteries was incompatible with the longitudinal nature of the protocol. After the experiments, the animals were allowed to recover and returned to their cages.

Single voxel ${ }^{1} H$ MRS. Multislice $\mathrm{T}_{2}$ weighted spin-echo imaging experiments (repetition time, $2 \mathrm{~s}$; echo time, $50 \mathrm{~ms}$; number 

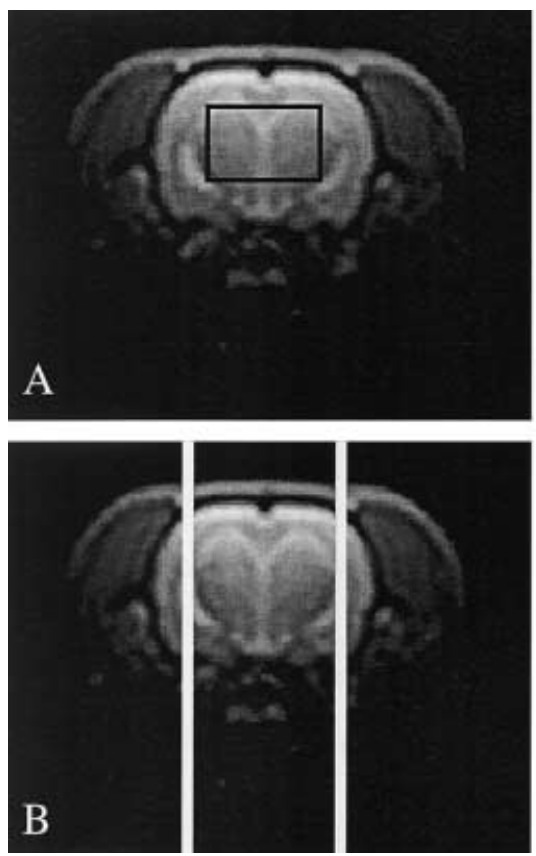

Fig. 1. Coronal and transversal $\mathrm{T}_{2}$-weighted images of a control rat brain, illustrating the localisation of the $240 \mu \mathrm{l}{ }^{1} \mathrm{H}$ MRS PRESS box (A,C; black box) and the localisation of the two-dimensional ISIS ${ }^{31} \mathrm{P}$ MRS column $(\mathrm{B}, \mathrm{C}$; white lines/box)

of acquisitions, $2 ; 15$ coronal slices of $1.2 \mathrm{~mm}$ thickness) was done to guide the positioning of the ${ }^{1} \mathrm{H}$ MRS volume of interest. A PRESS localisation technique [24] was used to define a $240 \mu \mathrm{l}$ voxel $\left(8 \times 5 \times 6 \mathrm{~mm}^{3}\right)$ centrally in the brain, including parts of the cortex, caudate-putamen, thalamus, hippocampus, corpus callosum and the ventricular system (Fig. 1). Anatomical landmarks determined the placement of the voxel. As multiple coronal slices guided the lateral position and depth of the voxel, the precision of tissue sampling was mainly determined by its rostro-caudal position. Owing to the partial volume effect, the $1.2 \mathrm{~mm}$ slice thickness thus allowed for a maximum variation in voxel placement in rostro-caudal direction of $0.6 \mathrm{~mm}$, leading to a maximum variation in tissue sampling of $10 \%$ between animals $(0.6 \mathrm{~mm} / 6 \mathrm{~mm})$. Given the relative limited variation of NAA, Cho and tCr concentrations in different brain areas, this $10 \%$ variation should not affect peak ratios [25].

Water suppression was achieved using CHESS pulses [26] and BISTRO, consisting of 32 frequency-selective radio frequency pulses with increasing amplitude [27]. Fully relaxed spectra were obtained, taking 64 transients with a relaxation time of $6 \mathrm{~s}$ and a total echo time of $144 \mathrm{~ms}$. At the final measurement, at 8 months, spectra were also acquired with four other echo times $(40,80,120$ and $180 \mathrm{~ms}$, with a relaxation time of $3 \mathrm{~s}$ ), to calculate the $\mathrm{T}_{2}$ relaxation time of NAA, $\mathrm{tCr}$ and Cho. The free induction decay was zero filled to $4 \mathrm{k}$ points, followed by exponential multiplication (4 Hz line-broadening). Spectra were obtained by Fourier transformation and were manually phased (zero and first order). The NAA resonance was assigned a chemical shift of $2.02 \mathrm{ppm}$. The relative amplitudes of the metabolite resonances were measured, using iterative fitting of the time domain signals with the variable projection method VARPRO [28]. Peak ratios of NAA:Cho,

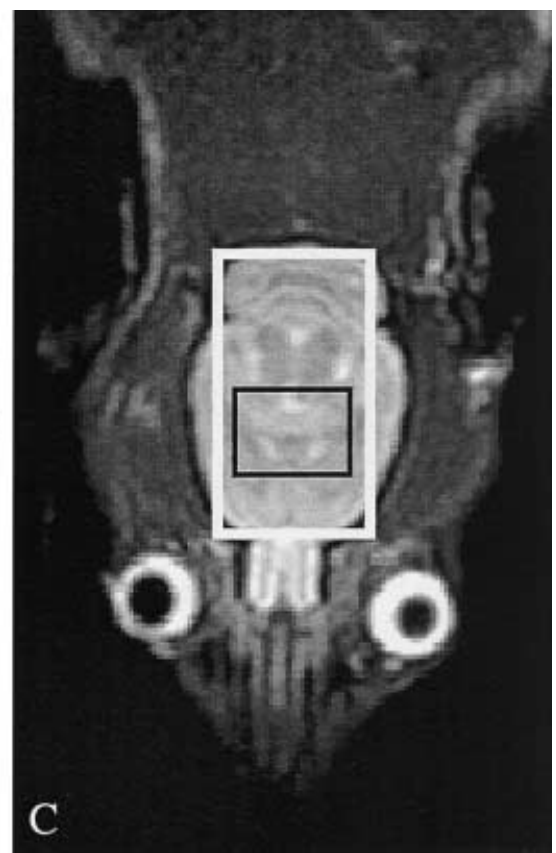

NAA:tCr and Cho:tCr were calculated. $\mathrm{T}_{2}$ relaxation times were calculated by fitting the decay of peak amplitudes at increasing echo time, assuming mono-exponential behaviour. Quantitative analysis of lactate concentrations was not feasible because normal cerebral lactate concentrations $(\sim 1 \mathrm{mmol} / \mathrm{l})$ are close to the detection limit for the present ${ }^{1} \mathrm{H}$ MRS technique [25]. Therefore, lactate peaks were assessed qualitatively. Lactate was considered detectable if a negative resonance at $1.33 \mathrm{ppm}$ clearly exceeded the noise level.

${ }^{31} \mathrm{P}$ Magnetic resonance spectroscopy. After the ${ }^{1} \mathrm{H}$ MRS experiments, the volume coil was detuned and the ${ }^{1} \mathrm{H}$ surface coil replaced by a ${ }^{31} \mathrm{P}$ coil. A transversal proton MR image was obtained to define a restricted column in the $\mathrm{z}(20 \mathrm{~mm})$ and $\mathrm{x}$ $(11 \mathrm{~mm})$ direction, perpendicular to the transversal image (Fig. 1). After localised shimming on the ${ }^{1} \mathrm{H}$ MR signal of water, a two-dimensional ISIS ${ }^{31} \mathrm{P}$ MRS experiment [29] was carried out on the column defined earlier. Pilot experiments showed that with the sensitivity of our surface coil and this two-dimensional localisation procedure, the ${ }^{31} \mathrm{P}$-spectrum is essentially derived from cerebral tissue and without contamination by phosphorous metabolites from extracranial (temporal and neck) muscles. For excitation an adiabatic half passage pulse was applied. Localisation was achieved by adiabatic full passage inversion pulses [25]. Transients $(n=128)$ were taken with a repetition time of $15 \mathrm{~s}$ to exclude differential saturation effects on the peak ratios. The free induction decay was zero filled to $4 \mathrm{k}$ points followed by exponential multiplication $(10$ $\mathrm{Hz}$ line broadening). After baseline-correction, the peaks of PCr, Pi and $\alpha$-ATP, $\beta$-ATP and $\gamma$-ATP were integrated in the frequency domain. The $\gamma$-ATP and $\beta$-ATP peaks were averaged. The PCr:Pi, PCr:ATP and ATP:Pi peak ratios were determined. Intracellular $\mathrm{pH}\left(\mathrm{pH}_{\mathrm{i}}\right)$ was calculated from the chemical shift of the Pi peak relative to the PCr peak [30].

Statistical analysis. Data are expressed as means \pm standard error of the mean (SEM), unless indicated otherwise. To evaluate the effect of diabetes, diabetic rats at 2 weeks were compared with baseline controls and diabetic rats at 8 months were compared with 8-month controls. Where there was homogeneity of variances (Levene test, $p>0.05$ ), group means 


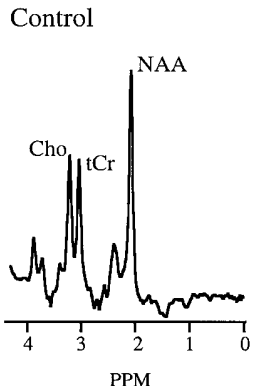

2 weeks diabetes

4 months diabetes
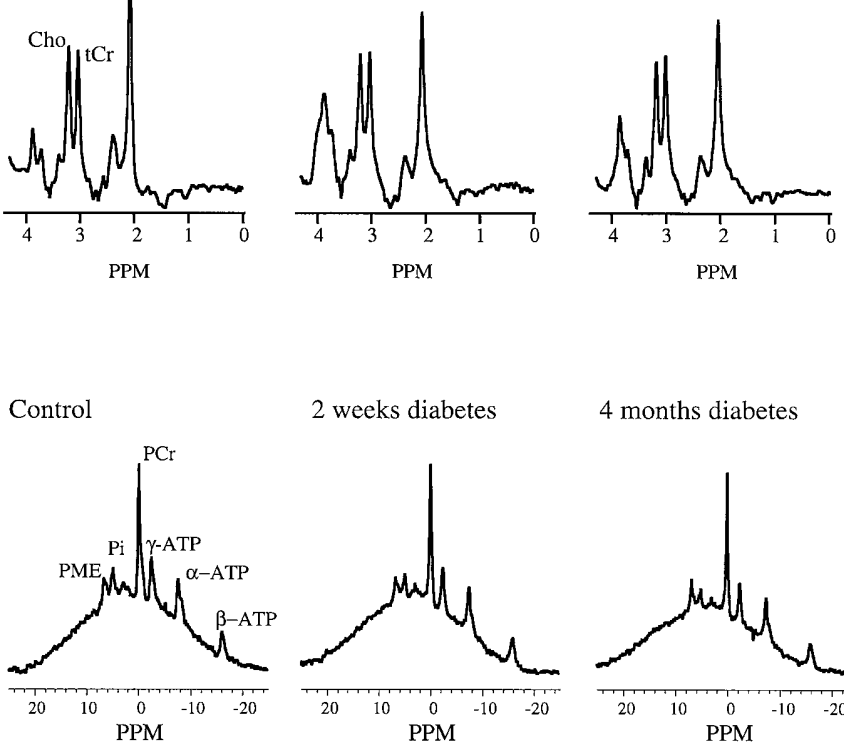

2 weeks diabetes

4 months diabetes
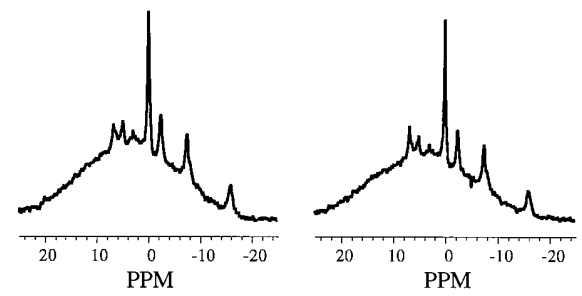

Fig. 2. Typical examples of single voxel PRESS ${ }^{1} \mathrm{H}$ MR spectra (upper panel) and ${ }^{31} \mathrm{P}$ MR spectra (lower panel) from baseline controls and diabetic rats 2 weeks and 4 months after streptozotocin injection. ${ }^{1} \mathrm{H}$ MR spectra were arbitrarily scaled on the total creatine $(\mathrm{tCr})$ peaks. The decrease in NAA:tCr and NAA:Cho peak ratios in diabetic rats at both time points, compared with control animals, is visible. Note the increase in amplitude of the resonance complex between 3.5 to 4 ppm in spectra from diabetic rats. ${ }^{31} \mathrm{P}$ MR spectra were scaled on the $\mathrm{PCr}$ peak. The relative peak amplitudes of the PCr, Pi, and ATP resonances do not change

were compared with a two-tailed $t$ test for independent samples. Because metabolite ratios in ${ }^{31} \mathrm{P}$ MRS were not distributed normally these data were analysed using Mann-Whitney U-test for nonparametric data. The effect of diabetes duration on ${ }^{1} \mathrm{H}$ MRS metabolite ratios and intracellular $\mathrm{pH}$ was tested with paired sample $t$ tests. The effects of diabetes duration on ${ }^{31} \mathrm{P}$ MRS metabolite ratios were assessed with Wilcoxon signed rank tests for nonparametric data.

\section{Results}

Body weight and blood glucose concentrations of the animals are presented in Table 1. Diabetic animals had significantly reduced body weights, which re- mained stable throughout the experiment. Blood glucose concentrations in diabetic animals were increased fourfold compared with controls.

${ }^{1}$ H Magnetic resonance spectroscopy (Table 2, Figure 2, 3). Typical ${ }^{1} \mathrm{H}$ MR spectra are presented in Figure 2. Peak ratios are presented in Figure 3. After 2 weeks and 8 months of diabetes, the NAA:tCr ratio was reduced by 14 and $23 \%$ compared with the corresponding controls $(p<0.001, p<0.005)$. The NAA:Cho ratio was decreased by 21 and $17 \%$ after 2 weeks and 8 months of diabetes compared with the respective control ratios $(p<0.001, p<0.005)$. There was no statistically significant effect of diabetes duration on metabolite ratios. The tCr:Cho ratio was not affected by diabetes. A small lactate resonance was detected in four out of eight controls at baseline and in two out of seven controls at 8 months. In diabetic animals a lactate resonance was detected in four out of seven rats after 2 weeks of diabetes, in one out of seven rats after 4 months of diabetes and in one out of seven rats after 8 months of diabetes.

We found that $T_{2}$ relaxation times at 8 months were similar in control and diabetic rats for NAA and Cho (Table 2). The $\mathrm{T}_{2}$ relaxation time of $\mathrm{tCr}$ was slightly but statistically significantly increased in the diabetic group (control $177 \pm 3$; diabetic $190 \pm 4 \mathrm{~ms}$; $\mathrm{p}<0.05)$. This $7 \%$ increase in relaxation time would at the most account for $6 \%$ of the reduction in NAA:tCr after 8 months of diabetes. Therefore the observed NAA:Cho and NAA:tCr reductions are not due to changes in NMR relaxation properties of these compounds.

In diabetic rats an increase in the signal intensity from 3.5 to 4 ppm was observed (Fig. 2). Several compounds, including inositol, glucose, glutamine and glutamate, could contribute to this signal [31]. Assessment of the levels of the individual compounds contributing to this resonance complex requires adapted MR protocols involving high-field strength, short echo times and/or spectral editing techniques [25].

${ }^{31}$ P Magnetic resonance spectroscopy (Figs. 2, 4). Typical ${ }^{31} \mathrm{P}$ MR spectra are presented in Figure 2. Peak ratios are presented in Figure 4. No statistically significant changes were observed in the PCr:ATP,

Table 1. Body weights, blood glucose

\begin{tabular}{|c|c|c|c|c|c|c|}
\hline & $\begin{array}{l}\text { Controls } \\
\text { baseline }\end{array}$ & $\begin{array}{l}\text { Controls } \\
8 \text { months }\end{array}$ & $\begin{array}{l}\text { DM } \\
\text { baseline }\end{array}$ & $\begin{array}{l}\text { DM } \\
2 \text { weeks }\end{array}$ & $\begin{array}{l}\text { DM } \\
4 \text { months }\end{array}$ & $\begin{array}{l}\text { DM } \\
8 \text { months }\end{array}$ \\
\hline Number of rats & 8 & 7 & 7 & 7 & 7 & 7 \\
\hline Body weight (g) & $356 \pm 5$ & $649 \pm 23$ & $353 \pm 8$ & $300 \pm 8^{a}$ & $309 \pm 15$ & $298 \pm 17^{\mathrm{a}}$ \\
\hline
\end{tabular}

Data are means \pm SEM and were analysed by two-tailed $t$ test for independent samples. Diabetic rats at 2 weeks were compared with baseline controls and diabetic rats at 8 months were compared with 8-month controls (significant differences between the groups are indicated: $\left.{ }^{\mathrm{a}} p<0.05\right)$. $\mathrm{DM}=$ diabetic 

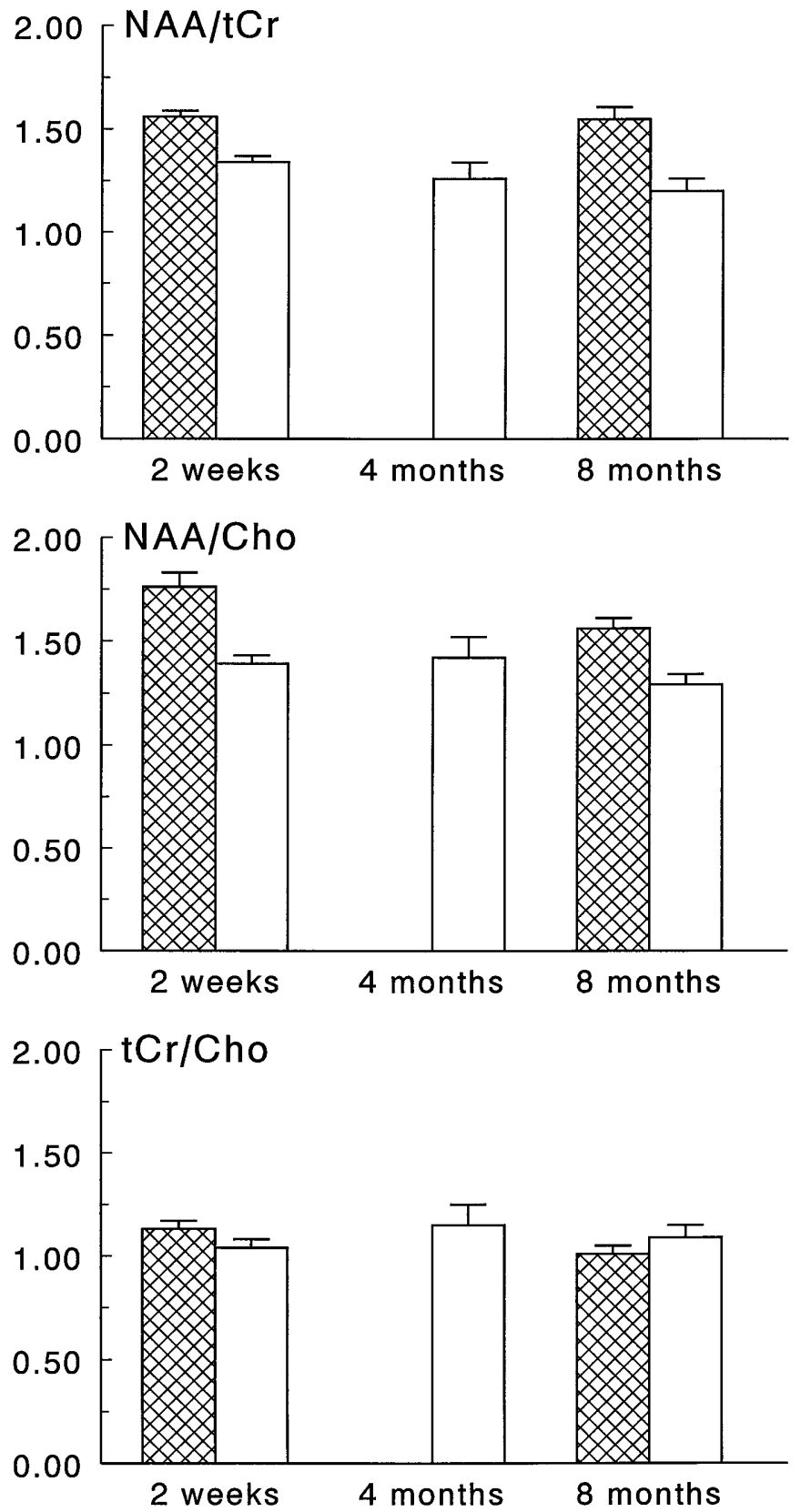

Fig. 3. ${ }^{1} \mathrm{H}$ MR metabolite ratios. Both the NAA:tCr and NAA:Cho ratio were significantly reduced after 2 weeks $(t$ test; both $p<0.001$ ) and 8 months (both $p<0.005$ ) of diabetes (open bars), compared with the respective baseline and 8month controls (hatched bars). There was no significant effect of diabetes duration on metabolite ratios (paired sample $t$ test). Data are means \pm SEM

PCr:Pi and ATP:Pi ratios after 2 weeks or 8 months of diabetes compared with the respective control values. There was no statistically significant effect of diabetes duration on peak ratios. The cerebral intracellular $\mathrm{pH}$ was similar in the three groups (control rats: baseline $7.18 \pm 0.02 ; 8$ months $7.13 \pm 0.02$; diabetic rats: 2 weeks $7.14 \pm 0.01 ; 4$ months $7.14 \pm 0.02$; 8 months $7.12 \pm 0.01)$.
Table 2. ${ }^{1} \mathrm{H}$ MRS: calculated $\mathrm{T}_{2}$ relaxation times for total creatine, choline and $N$-acetylaspartate at 8 months

\begin{tabular}{lll}
\hline & Controls & Diabetic \\
\hline $\mathrm{T}_{2}$ total creatine & $177 \pm 3$ & $190 \pm 4^{\mathrm{a}}$ \\
$\mathrm{T}_{2}$ choline & $317 \pm 11$ & $303 \pm 13$ \\
$\mathrm{~T}_{2} \mathrm{~N}$-acetylaspartate & $358 \pm 16$ & $358 \pm 13$ \\
\hline
\end{tabular}

Data are means \pm SEM and were analysed by two-tailed $t$ test for independent samples. Diabetic rats at 8 months were compared with 8-month controls (significant differences between the groups are indicated: $\left.{ }^{a} p<0.05\right)$

\section{Discussion}

Cerebral metabolism in diabetic rats was examined longitudinally with in vivo ${ }^{31} \mathrm{P}$ and ${ }^{1} \mathrm{H}$ MRS. No changes in cerebral high-energy phosphate metabolites were observed compared with controls up to 8 months after diabetes induction. Moreover, in the brain of diabetic rats lactate peaks were detected as frequently as in controls and the intracellular $\mathrm{pH}$ was unaffected. Diabetes seems not to have affected the energy status of the brain. It did lead to a statistically significant decrease in the NAA:tCr and NAA: Cho ratios at all time points studied. Notably, reduced cerebral NAA resonances have also been shown in a small group of diabetic patients [32]. Although in our study the amount of NAA was assessed relative to that of $\mathrm{tCr}$ and $\mathrm{Cho}$, the data strongly suggest that diabetes reduces the concentration of NAA in the brain. The alternative explanation, that diabetes simultaneously increased the concentrations of $\mathrm{tCr}$ and of Cho, is unlikely. The $\mathrm{tCr}$ peak is believed to be relatively insensitive to tissue pathology, and is often used as a reference resonance for the measurement of relative changes in NAA or Cho or both [21]. The Cho peak reflects cerebral choline stores. Choline-containing compounds are involved in membrane synthesis and degradation [21], and increased concentrations of choline are associated with conditions such as brain tumours and demyelinating disease [20]. Even in peripheral nerves of STZ-diabetic rats, in which pathological changes are generally more pronounced than in the brain, demyelination does not occur as early as after 2 weeks of diabetes [33].

The metabolite, NAA, is found in high concentrations $(8-10 \mathrm{mmol} / \mathrm{l})$ only in the brain $[21,20]$. Because it is present largely, if not entirely, in neurones [21], the NAA resonance is often used as a neuronal marker, although its physiological role is largely not known $[21,20]$. A relative decrease in cerebral NAA levels has now been reported in such disorders as stroke, Alzheimer's disease and multiple sclerosis and has been attributed to neuronal loss [21, 20]. More recently reductions in cerebral NAA were, however, shown to be potentially reversible [34, 35], indicating that NAA levels not only reflect neuronal 

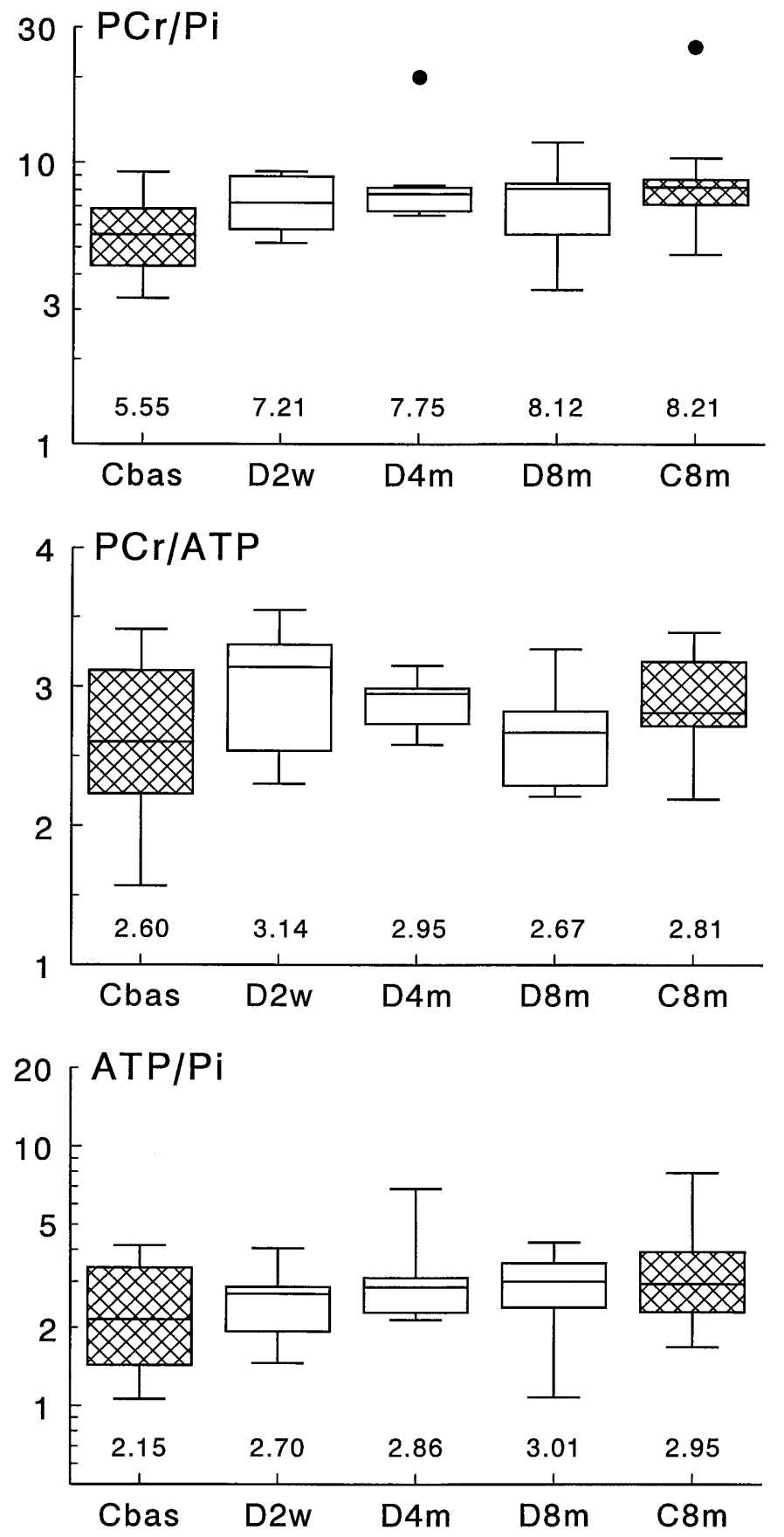

Fig. 4. ${ }^{31} \mathrm{P}$ MR metabolite ratios. Boxes present quartile ranges with median. Whiskers indicate range. Extreme outliers (more than three times interquartile range) are indicated ( $)$. Median values are printed below boxes. No significant differences were detected between 2-week diabetic rats and baseline controls and between 8-month diabetic rats and 8-month controls (Mann-Whitney U test). There was no significant effect of diabetes duration on metabolite ratios (Wilcoxon signed rank test)

density and viability but can also be used as a dynamic marker of neuronal metabolic (dys)function and integrity [34]. This latter interpretation would be in line with our observation that NAA ratios in the brain of STZ-diabetic rats were reduced well before functional and structural abnormalities are known to occur in this model. Deficits in learning and memory and in signal transduction, for example, become detectable at 2 to 3 months after diabetes induction and develop gradually thereafter $[9,6,7]$. Whether reductions in NAA, as an early marker of neuronal dysfunction in STZ-diabetes, are causally related to the behavioural, neurophysiological and structural cerebral changes that occur later on is not known.

Phosphocreatine is a high-energy phosphorus compound that serves as an energy buffer in the creatinekinase-mediated ATP:ADP equilibrium. Under (moderate) ischaemic or hypoxic conditions a fall in cerebral ATP is preceded by a reduction in $\mathrm{PCr}$ and a rise in lactate $[36,37]$. In our study the PCr:ATP ratio was unaffected up to 8 months after diabetes induction. In addition, cerebral lactate was detected in a similar percentage of diabetic and control animals, indicating that diabetes did not lead to an increased dependence of the brain on anaerobic glycolysis. It should be noted, however, that with the present technique normal cerebral lactate concentrations are near the detection threshold for in vivo MRS in rats, which limits the assessment of subtle changes. Higher magnetic field strength and shorter echo times are required for quantitative measurements of normal or slightly increased cerebral lactate concentrations [38]. Our observations are in line with the baseline findings of an earlier in vivo ${ }^{31} \mathrm{P}$ MRS study, which examined the effects of acute ischaemia in $\mathrm{BB} /$ Wor diabetic rats [39]. In that study $\mathrm{PCr}$ concentrations in the brain of 4-month diabetic $\mathrm{BB} /$ Wor rats before induction of ischaemia were similar to controls.

Earlier studies on cerebral blood flow in diabetic rats have provided results that seem to be inconsistent [16]. It is likely, however, that differences in the techniques used for assessing blood flow are the major source of this inconsistency. For example, studies that assessed blood flow indirectly, by measuring erythrocyte velocity in cortical arterioles [40] or venous outflow [41] reported increases in cerebral blood flow. In contrast, studies that assessed blood flow at the tissue level, by measuring the cerebral uptake of tracers like $\left[{ }^{14} \mathrm{C}\right]$ iodoantipyrine in awake or anaesthetized rats, consistently report flow reductions of 10 to $15 \%$ during the first month of diabetes $[12,13]$ and 10 to $30 \%$ after 4 months of diabetes $[42,14]$, with some degree of regional variation. Structural changes in the vasculature, including basement membrane thickening [15] could further impede energy delivery to the brain. Our data indicate that these vascular abnormalities are of insufficient magnitude to compromise the energy status of the brain to a level detectable with MRS. This does not rule out involvement of vascular disturbances in cerebral dysfunction in diabetes. The sensitivity of MRS might not be insufficient to detect subtle regional variations. More importantly, energy consumption is 
known to be reduced in the brain of diabetic rats [14], which could counterbalance an impaired energy delivery, thus preserving the energy status of the brain. Moreover, studies on the effects of mild to moderate hypoxia in non-diabetic animals showed that changes in cognitive functions and the electroencephalogram can occur at oxygen levels at which the $\mathrm{PCr}$ and ATP concentrations in the brain are still in the normal range [37]. The functional deficits at these moderate levels of tissue hypoxia were suggested to be the consequence of oxygen-dependent changes in the metabolism of neurotransmitters rather than the effect of a failure in ATP and PCr production [37]. The threshold at which chronic reductions in cerebral blood flow lead to reductions in PCr and ATP is not exactly known but seems to exceed the $10-30 \%$ flow reduction that can be expected to occur in the brain of STZ-diabetic rats $[12,13,14,42]$. In comparison, in peripheral nerves of diabetic rats, where blood flow is reduced by approximately $50 \%$ [43, 44], increased lactate and decreased PCr concentrations have been observed $[22,45,46]$. The reductions in $\mathrm{PCr}$ are still relatively small and have not been found consistently $[47,46]$. In the superior cervical ganglion, which like the brain has a higher metabolic rate, blood flow is reduced by $45 \%$ and $\mathrm{PCr}$ is reduced by $38 \%$ in STZ-diabetic rats [48, 46]. Likewise, in the retina of diabetic rabbits ATP concentrations can also be reduced [49], but it should be noted that ATP reductions in the retina have not been found consistently [50] and much controversy still exists about the nature of blood flow changes in the retina [51].

The pathogenesis of cerebral dysfunction in diabetes seems to be a multifactorial process [1]. Therefore, in addition to cerebrovascular changes other factors, such as aberrant glucose metabolism in glia and neurones, are likely to affect the brain. Notably, increased glucose concentrations in the brain of STZ-diabetic rats were found to be associated with oxidative damage [52] and accumulation of sorbitol, fructose and advanced glycation end products $[53,54,55]$, albeit to a lesser extent than in peripheral nerves. These metabolic changes are paralleled by a reduction in $\mathrm{Na}^{+}$, $\mathrm{K}^{+}$-ATPase activity in brain homogenates [56], which could account for a part of the reduced energy consumption in the brain of diabetic rats [14].

In conclusion, in vivo ${ }^{31} \mathrm{P}$ and ${ }^{1} \mathrm{H}$ MRS of the brain of diabetic rats showed no alterations in either highenergy phosphate metabolite ratios or lactate resonances after a diabetes duration of 2 weeks to 8 months. Therefore, reductions of cerebral blood flow in STZ-diabetic rats do not seem to affect the energy status of the brain. In vivo ${ }^{1} \mathrm{H}$ MRS showed a relative reduction in NAA in the brain of STZ-diabetic rats, which could present a marker for early metabolic or functional abnormalities in cerebral neurones in diabetes.

\section{References}

1. Biessels GJ, Kappelle AC, Bravenboer B, Erkelens DW, Gispen WH (1994) Cerebral function in diabetes mellitus. Diabetologia 37: 643-650

2. Ryan CM (1988) Neurobehavioral complications of type I diabetes. Examination of possible risk factors. Diabetes Care 11: 86-93

3. Stewart R, Liolitsa D (1999) Type 2 diabetes mellitus, cognitive impairment and dementia. Diabet Med 16: 93-112

4. Ott A, Stolk RP, Van Harskamp F, Pols HA, Hofman A, Breteler MM (1999) Diabetes mellitus and the risk of dementia: The Rotterdam Study. Neurology 53: 1937-1942

5. Biessels GJ, Kamal A, Ramakers GM et al. (1996) Place learning and hippocampal synaptic plasticity in streptozotocin-induced diabetic rats. Diabetes 45: 1259-1266

6. Biessels GJ, Cristino NA, Rutten G, Hamers FPT, Erkelens DW, Gispen WH (1999) Neurophysiological changes in the central and peripheral nervous system of streptozotocin-diabetic rats: course of development and effects of insulin treatment. Brain 122: 757-768

7. Morano S, Sensi M, Di Gregorio S et al. (1996) Peripheral, but not central, nervous system abnormalities are reversed by pancreatic islet transplantation in diabetic Lewis rats. Eur J Neurosci 8: 1117-1123

8. Jakobsen J, Sidenius P, Gundersen HJ, Osterby R (1987) Quantitative changes of cerebral neocortical structure in insulin-treated long-term streptozocin-induced diabetes in rats. Diabetes 36: 597-601

9. Biessels GJ, Kamal A, Urban IJ, Spruijt BM, Erkelens DW, Gispen WH (1998) Water maze learning and hippocampal synaptic plasticity in streptozotocin-diabetic rats: effects of insulin treatment. Brain Res 800: 125-135

10. Schnedl WJ, Ferber S, Johnson JH, Newgard CB (1994) STZ transport and cytotoxicity. Specific enhancement in GLUT2- expressing cells. Diabetes 43: 1326-1333

11. Simpson IA, Appel NM, Hokari M et al. (1999) Bloodbrain barrier glucose transporter: effects of hypo- and hyperglycemia revisited. J Neurochem 72: 238-247

12. Harik SI, LaManna JC (1988) Vascular perfusion and blood-brain glucose transport in acute and chronic hyperglycemia. J Neurochem 51: 1924-1929

13. Duckrow RB, Beard DC, Brennan RW (1987) Regional cerebral blood flow decreases during chronic and acute hyperglycemia. Stroke 18: 52-58

14. Jakobsen J, Nedergaard M, Aarslew Jensen M, Diemer NH (1990) Regional brain glucose metabolism and blood flow in streptozocin- induced diabetic rats. Diabetes 39: 437-440

15. Junker U, Jaggi C, Bestetti G, Rossi GL (1985) Basement membrane of hypothalamus and cortex capillaries from normotensive and spontaneously hypertensive rats with steptozotocin-induced diabetes. Acta Neuropathol (Berl) 65: 202-208

16. Mankovsky BN, Metzger BE, Molitch ME, Biller J (1997) Cerebrovascular disorders in patients with diabetes mellitus. J Diabetes Complications 10: 228-242

17. Lanier WL, Hofer RE, Gallagher WJ (1996) Metabolism of glucose, glycogen, and high-energy phosphates during transient forebrain ischemia in diabetic rats: effect of insulin treatment. Anesthesiology 84: 917-925

18. Wagner SR,Lanier WL (1994) Metabolism of glucose, glycogen, and high-energy phosphates during complete cerebral ischemia. A comparison of normoglycemic, chronically hyperglycemic diabetic, and acutely hyperglycemic nondiabetic rats. Anesthesiology 81: 1516-1526 
19. Mans AM, DeJoseph MR, Davis DW, Hawkins RA (1988) Brain energy metabolism in streptozotocin-diabetes. Biochem J 249: 57-62

20. Vion Dury J, Meyerhoff DJ, Cozzone PJ, Weiner MW (1994) What might be the impact on neurology of the analysis of brain metabolism by in vivo magnetic resonance spectroscopy? J Neurol 241: 354-371

21. Miller BL (1991) A review of chemical issues in ${ }^{1} \mathrm{H}$ NMR spectroscopy: $N$-acetyl-L-aspartate, creatine and choline. NMR Biomed 4: 47-52

22. Obrosova IG, Fathallah L, Lang HJ, Greene DA (1999) Evaluation of a sorbitol dehydrogenase inhibitor on diabetic peripheral nerve metabolism: a prevention study. Diabetologia 42: 1187-1194

23. Shafrir E (1997) Diabetes in animals: contribution to the understanding of diabetes by study of its etiopathology in animal models. In: Porte JR, D, Sherwin RS (eds) Ellenberg and Rifkin's diabetes mellitus; theory and practice. 5 edn. Appleton \& Lange, Stamford, pp 301-348

24. Bottomley PA (1987) Selective volume method for performing spatial localization in NMR spectroscopy in vivo. Ann NY Acad Sci 508: 333-348

25. De Graaf RA (1998) In vivo NMR spectroscopy: principles and techniques. John Wiley \& Sons Ltd., Chicester

26. Haase A, Frahm J, Hänicke W, Matthaei D (1985) 1H NMR chemical shift selective (CHESS) imaging. Phys Med Biol 30: 341-344

27. De Graaf RA, Luo Y, Garwood M, Nicolay K (1996) B1-insensitive, single-shot localization and water suppression. J Magn Reson 113: 35-45

28. de Beer R, van Osmondt D, Pijnappel WWF, van der Veen JWC (1988) Quantitative analysis of magnetic resonance signals in the time domain. Isr J Chem 28: 249-261

29. Ordidge RJ, Conelly A, Lohman JAB (1986) Image-selected in vivo spectroscopy (ISIS). A new technique for spatially selective NMR spectroscopy. J Magn Reson 66: 283-294

30. Petroff OAC, Prichard JW, Behar KL, Alger JR, den Hollander JA, Shulman RG (1985) Cerebral intracellualr $\mathrm{pH}$ by ${ }^{31} \mathrm{P}$ nuclear magnetic resonance spectroscopy. Neurology 35: 781-788

31. Kreis R, Ross BD (1992) Cerebral metabolic disturbances in patients with subacute and chronic diabetes mellitus: detection with proton MR spectroscopy. Radiology 184: 123-130

32. Kreis R, Ross BD (1992) Cerebral metabolic disturbances in patients with subacute and chronic diabetes mellitus: detection with proton MR spectroscopy. Radiology 184: 123-130

33. Yagihashi S (1995) Pathology and pathogenetic mechanisms of diabetic neuropathy. Diabetes Metab Rev 11: 193-225

34. Brulatout S, Meric P, Loubinoux I et al. (1996) A one-dimensional (proton and phosphorus) and two-dimensional (proton) in vivo NMR spectroscopic study of reversible global cerebral ischemia. J Neurochem 66: 2491-2499

35. De Stefano N, Matthews PM, Arnold DL (1995) Reversible decreases in $\mathrm{N}$-acetylaspartate after acute brain injury. Magn Reson Med 34: 721-727

36. Salford LG, Plum F, Siesjo BK (1973) Graded hypoxia oligemia in rat brain. Arch Neurol 29: 227-233

37. Gibson GE, Pulsinelli W, Blass JP, Duffy TE (1981) Brain dysfunction in mild to moderate hypoxia. Am J Med 70: 1247-1254

38. Pfeuffer J, Tkac I, Gruetter R (2000) Extracellular-intracellular distribution of glucose and lactate in the rat brain assessed noninvasively by diffusion-weighted $1 \mathrm{H}$ nuclear magnetic resonance spectroscopy in vivo. J Cereb Blood Flow Metab 20: 736-746
39. Sutherland GR, Peeling J, Sutherland E et al. (1992) Forebrain ischemia in diabetic and nondiabetic BB rats studied with $31 \mathrm{P}$ magnetic resonance spectroscopy. Diabetes 41: 1328-1334

40. Rubin MJ, Bohlen HG (1985) Cerebral vascular autoregulation of blood flow and tissue $\mathrm{PO}_{2}$ in diabetic rats. Am J Physiol 249: H540-H546

41. Simpson RE 3rd, Phillis JW, Buchannan J (1990) A comparison of cerebral blood flow during basal, hypotensive, hypoxic and hypercapnic conditions between normal and streptozotocin diabetic rats. Brain Res 531: 136-142

42. Knudsen GM, Gobel U, Paulson OB, Kuschinsky W (1991) Regional density of perfused capillaries and cerebral blood flow in untreated short-term and long-term streptozotocin diabetes. J Cereb Blood Flow Metab 11: 361-365

43. Tuck RR, Schmelzer JD, Low PA (1984) Endoneurial blood flow and oxygen tension in the sciatic nerves of rats with experimental diabetic neuropathy. Brain 107: 935-950

44. Cameron NE, Cotter MA (1994) The relationship of vascular changes to metabolic factors in diabetes mellitus and their role in the development of peripheral nerve complications. Diabetes Metab Rev 10: 189-224

45. Low PA, Ward K, Schmelzer JD, Brimijoin S (1985) Ischemic conduction failure and energy metabolism in experimental diabetic neuropathy. Am J Physiol 248: E457-E462

46. Kishi Y, Schmelzer JD, Yao JK et al. (1999) Alpha-lipoic acid: effect on glucose uptake, sorbitol pathway, and energy metabolism in experimental diabetic neuropathy. Diabetes 48: 2045-2051

47. Yasuda H, Sonobe M, Yamashita M et al. (1989) Effect of prostaglandin E1 analogue TFC 612 on diabetic neuropathy in streptozocin-induced diabetic rats. Comparison with aldose reductase inhibitor ONO 2235. Diabetes 38: 832-838

48. Sasaki H, Schmelzer JD, Zollman PJ, Low PA (1997) Neuropathology and blood flow of nerve, spinal roots and dorsal root ganglia in longstanding diabetic rats. Acta Neuropathol (Berl ) 93: 118-128

49. Drago F, La Manna C, Emmi I, Marino A (1998) Effects of sulfinpyrazone on retinal damage induced by experimental diabetes mellitus in rabbits. Pharmacol Res 38: 97-100

50. Kern TS, Kowluru RA, Engerman RL (1994) Abnormalities of retinal metabolism in diabetes or galactosemia: ATPases and glutathione. Invest Ophthalmol Vis Sci 35: 2962-2967

51. Schmetterer L, Wolzt M (1999) Ocular blood flow and associated functional deviations in diabetic retinopathy. Diabetologia 42: 387-405

52. Kumar JS, Menon VP (1993) Effect of diabetes on levels of lipid peroxides and glycolipids in rat brain. Metabolism 42: 1435-1439

53. Vlassara H, Brownlee M, Cerami A (1983) Excessive non enzymatic glycosylation of peripheral and central nervous system myelin components in diabetic rats. Diabetes 32 : 670-674

54. Sredy J, Sawicki DR, Notvest RR (1991) Polyol pathway activity in nervous tissues of diabetic and galactose-fed rats: effect of dietary galactose withdrawal or tolrestat intervention therapy. J Diabetes Complications 5: 42-47

55. Knudsen GM, Jakobsen J, Barry DI, Compton AM, Tomlinson DR (1989) Myo-inositol normalizes decreased sodium permeability of the blood-brain barrier in streptozotocin diabetes. Neuroscience 29: 773-777

56. Leong SF, Leung TK (1991) Diabetes induced by streptozotocin causes reduced Na-K ATPase in the brain. Neurochem Res 16: 1161-1165 